\title{
Doping-induced phase transition enables better electrocatalysts
}

\author{
Xiao Zhang, Zhuangchai Lai and Hua Zhang*
}

Switching from fossil-fuel economy to hydrogen economy requires efficient catalysts to facilitate several fundamental electrochemical reactions, including oxygen evolution reaction (OER), hydrogen evolution reaction (HER), oxygen reduction reaction (ORR), and hydrogen oxidation reaction (HOR). At present, platinum-group materials are still considered as the state-of-the-art catalysts, which, however, are rare and expensive, resulting in tremendous restrictions to wide usage. The future of hydrogen economy demands the design of cost-effective and highly active catalysts that are geologically abundant, particularly for affordable and scalable device systems. As for non-platinum-group electrocatalysts, strategies to enhance their catalytic performances include controlling their size, morphology and composition, creating defects and strains, as well as modulating the van der Waals interactions and crystal phases, etc. [1,2]. Although remarkable progress has been achieved, innovations in catalyst design that aim to further promote catalytic activities are still highly desired.

In a recent article in Nature Communications, $\mathrm{Yu}$ and co-workers [3] from the University of Science and Technology of China reported the first experimental observation of phosphorus (P)-doping-induced phase transition from the cubic $\mathrm{CoSe}_{2}\left(c-\mathrm{CoSe}_{2}\right)$ to orthorhombic $\operatorname{CoSe}_{2}\left(o-\mathrm{CoSe}_{2} \mid \mathrm{P}\right)$, which exhibits highly efficient electrocatalytic HER activity. The obtained $o$-CoSe $e_{2} \mid \mathrm{P}$ catalyst possesses belt-like morphology (Fig. 1a), similar to the original $c-\mathrm{CoSe}_{2}$. It is noted that phase change from $o$-CoSe $e_{2}$ to $c$-CoSe $e_{2}$ can be easily realized through a thermal synthetic route [4]. However, the reverse phase transformation has not been achieved due to the higher thermodynamical stability of the cubic phase. $\mathrm{Yu}$ and co-workers [3] found that the introduction of P-doping can achieve the phase change from $c-\mathrm{CoSe}_{2}$ to $o-\mathrm{CoSe}_{2} \mid \mathrm{P}$. The new phase transition process was monitored by various characterization techniques, such as temperature- dependent X-ray diffraction (Fig. 1b) and Raman spectroscopy (Fig. 1c). Based on these experimental results, the researchers proposed the phase change mechanism. Annealing $c$ - $\mathrm{CoSe}_{2}$ at $400^{\circ} \mathrm{C}$ results in the loss of some Se atoms, thus leaving vacancy defects, which were then quickly occupied by the in situ generated $\mathrm{P}$ atoms. Because of the weaker electronegativity of $\mathrm{P}$ compared to Se, its doping induces the formation of stronger Co-P interactions and elongated $\mathrm{Se}-\mathrm{Se}(\mathrm{P})$ electron pairs. Such new bond reconstructions allow for tuning electrons in $d$ orbitals on $\mathrm{Co}$, which was regarded as the driving force for rotation of $\mathrm{Se}-\mathrm{Se}(\mathrm{P})$ pairs, thus forming the $o$-CoSe $2 \mid \mathrm{P}$ (Fig. 1d).

As known, both the HER and HOR kinetics in alkaline electrolyte are significantly slower than that in acidic conditions on platinum, limiting the further development of alkaline electrolysers and alkaline fuel cells [5]. In their work, $\mathrm{Yu}$ and co-workers combined the electrochemical experiments and theoretical calculations to explore the capability of HER electrocatalysis using $o-\mathrm{CoSe}_{2} \mid \mathrm{P}$ in alkaline solution. Thanks to the favorable electronic structure and local coordination environment created by the P-doping-induced phase transition strategy (Fig. 1e), the new $o$-CoSe $2 \mid \mathrm{P}$ only requires an overpotential of $104 \mathrm{mV}$ at $10 \mathrm{~mA} \mathrm{~cm}^{-2}$ in $1 \mathrm{~mol} \mathrm{~L}^{-1} \mathrm{KOH}$, with a small onset potential of $31 \mathrm{mV}$, which is better than those of previously reported electrocatalysts (Fig. 1f). Their results provide a strategy for preparing highly efficient electrocatalysts through the element-doping-induced phase transition, which might be used for other materials.

Received 30 July 2018; accepted 31 July 2018; published online 14 August 2018

1 Mistry H, Varela AS, Kühl S, et al. Nanostructured electrocatalysts with tunable activity and selectivity. Nat Rev Mater, 2016, 1: 16009

2 Seh ZW, Kibsgaard J, Dickens CF, et al. Combining theory and experiment in electrocatalysis: insights into materials design. Sci-

School of Materials Science and Engineering, Nanyang Technological University, N4.1-02-22, Block N4.1, 50 Nanyang Avenue, 639798, Singapore

*Email: HZhang@ntu.edu.sg 

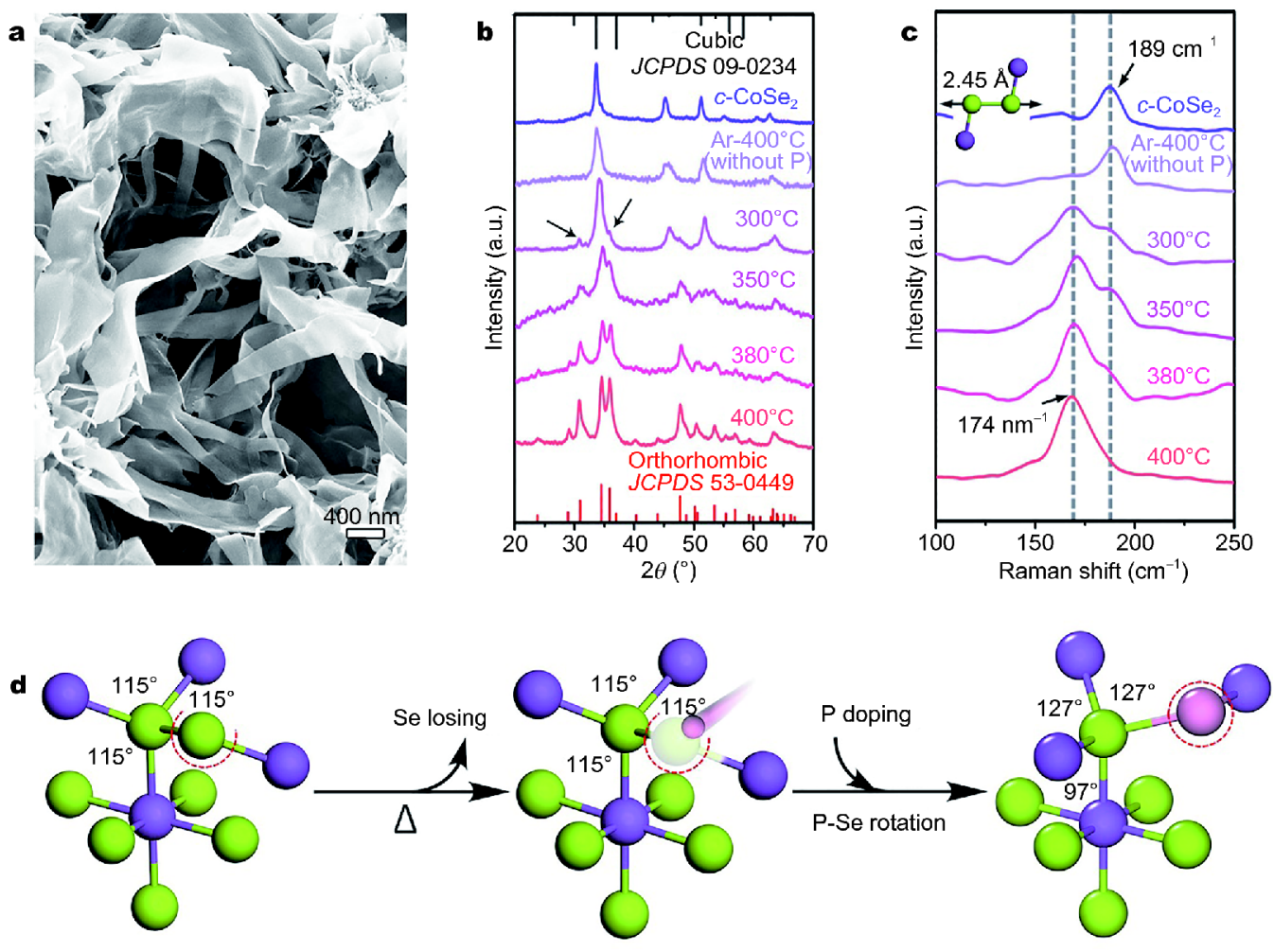

e

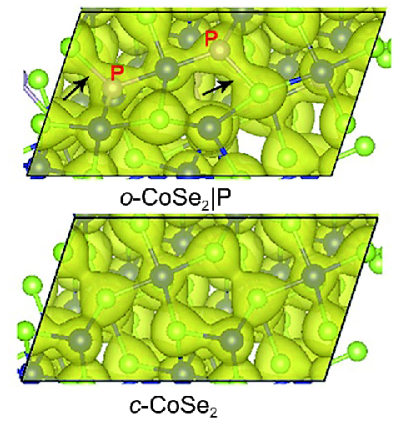

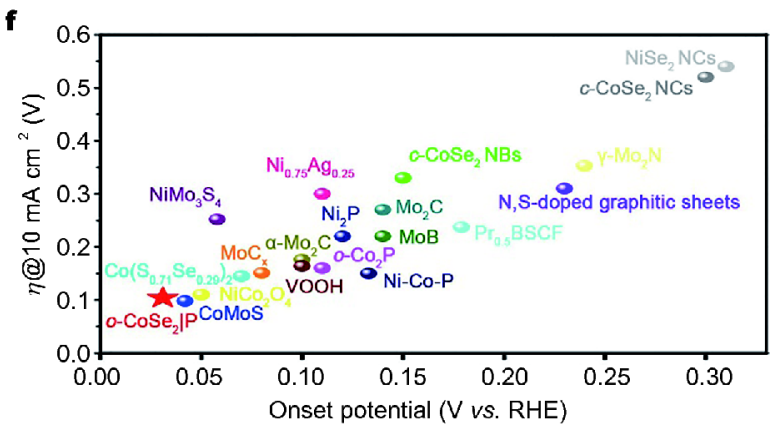

Figure 1 (a) SEM image of the obtained $o$-CoSe $2 \mid \mathrm{P}$ nanobelts. (b) Temperature-dependent XRD patterns and (c) Raman spectra revealing the phase change from $c$ - $\mathrm{CoSe}_{2}$ to $o$-CoSe $2 \mid \mathrm{P}$. (d) Schematic illustration of the P-doping-induced phase transition process from $c$-CoSe $e_{2}$ to $o$-CoSe $2 \mid \mathrm{P}$. (e) Calculated charge density distribution for $o$-CoSe $2 \mid \mathrm{P}$ (top) and $c$-CoSe $e_{2}$ (bottom) catalysts. (f) Comparison of the onset potentials and overpotentials $(\eta)$ for various non-noble-metal HER catalysts in alkaline electrolytes. The data were obtained from previously published literatures. Reprinted with permission from Ref. [3].

ence, 2017, 355: eaad4998

3 Zheng YR, Wu P, Gao MR, et al. Doping-induced structural phase transition in cobalt diselenide enables enhanced hydrogen evolution catalysis. Nat Commun, 2018, 9: 2533

4 Chen $\mathrm{P}, \mathrm{Xu} \mathrm{K}$, Tao S, et al. Phase-transformation engineering in cobalt diselenide realizing enhanced catalytic activity for hydrogen evolution in an alkaline medium. Adv Mater, 2016, 28: 7527-7532

5 Sheng W, Zhuang Z, Gao M, et al. Correlating hydrogen oxidation and evolution activity on platinum at different $\mathrm{pH}$ with measured hydrogen binding energy. Nat Commun, 2015, 6: 5848 